\title{
Romosozumab, clinical trials, and real-world care of patients with osteoporosis
}

\author{
E. Michael Lewiecki
}

New Mexico Clinical Research \& Osteoporosis Center, Albuquerque, NM, USA

Correspondence to: E. Michael Lewiecki, MD. New Mexico Clinical Research \& Osteoporosis Center, 300 Oak St. NE, Albuquerque, NM 87106, USA. Email mlewiecki@gmail.com.

Provenance and Peer Review: This article was commissioned and reviewed by the Academic Editor Dr. Yusheng Li (Department of Orthopedics, Xiangya Hospital, Central South University, Changsha, China).

Comment on: McClung MR, Brown JP, Diez-Perez A, et al. Effects of 24 Months of Treatment With Romosozumab Followed by 12 Months of Denosumab or Placebo in Postmenopausal Women With Low Bone Mineral Density: A Randomized, Double-Blind, Phase 2, Parallel Group Study. J Bone Miner Res 2018;33:1397-406.

Submitted Mar 02, 2020. Accepted for publication Mar 13, 2020.

doi: $10.21037 /$ atm.2020.03.196

View this article at: http://dx.doi.org/10.21037/atm.2020.03.196

Osteoporosis is a global public health concern with serious personal consequences and high societal costs due to fractures (1). Medications are available that have been proven to reduce fracture risk with a wide margin of safety, providing opportunities to diminish the burden of osteoporotic fractures. Many of these medications are available in low cost generic formulations. All approved agents work by modulating bone remodeling, the physiologic process by which bone is continually resorbed by osteoclasts and formed by osteoblasts. In postmenopausal osteoporosis, bone remodeling is accelerated, with an imbalance of resorption greater than formation, resulting in a measurable decrease of bone mineral density (BMD) and degradation of bone structure over time, with loss of bone strength and increase of fracture risk. In what seems like a paradox, osteoporosis can be treated with drugs that inhibit bone remodeling ("antiresorptives") and others that stimulate bone remodeling ("anabolics" or "bone building"). The critical factor for achieving a beneficial skeletal effect is the balance between bone resorption and formation. When treatment results in bone formation that exceeds resorption, there is a net accrual of bone, regardless of the direction of the changes. Antiresorptive agents (e.g., bisphosphonates, denosumab) reduce bone resorption more than they reduce formation, resulting in an increase of BMD, while anabolic agents [e.g., parathyroid hormone (PTH) derivativesteriparatide, abaloparatide], increase bone formation more than they increase resorption, also increasing BMD. Anabolic agents can improve the structure of bone, making them an especially attractive choice for treatment of patients at very high risk of fracture, when degradation of bone structure is often advanced. Romosozumab, described below, is an anabolic agent with a different mechanism of action than the PTH-derived compounds. It is the latest osteoporosis drug to receive regulatory approval. Given the enormous costs of development of osteoporosis treatments and the current regulatory hurdles, it may be many years before another drug to reduce fracture risk is approved.

It is informative to examine what has been learned with studies of the newest treatment for osteoporosis and how this knowledge can be applied to current concepts for the management of patients in clinical practice (Table 1).

\section{Romosozumab}

Romosozumab is a humanized monoclonal antibody to sclerostin, an endogenous cytokine that inhibits bone formation and stimulates bone resorption. By blocking sclerostin, romosozumab has "dual effect" of increasing bone formation and decreasing bone resorption. The unique mechanism of action of romosozumab distinguishes this medication from the other anabolic agents, teriparatide and abaloparatide, expanding the options for individualizing treatment decisions for patients at high risk of fracture. 
Table 1 Concepts for treating patients with osteoporosis. These are considerations for patient management derived from the best available medical evidence. Treatment decisions should be individualized for each patient according to all available clinical information

\begin{tabular}{|c|c|}
\hline Concepts & Comments \\
\hline $\begin{array}{l}\text { Osteoporosis drugs stop working } \\
\text { when they are stopped }\end{array}$ & $\begin{array}{l}\text { Non-bisphosphonate drugs rapidly lose their therapeutic effects when stopped; bisphosphonates } \\
\text { have a slower offset of effect when stopped due to long skeletal half-life }\end{array}$ \\
\hline $\begin{array}{l}\text { Treated osteoporosis is still } \\
\text { osteoporosis }\end{array}$ & $\begin{array}{l}\text { There is no cure for osteoporosis. When treatment of a patient results in T-score }>-2.5 \text {, the patient } \\
\text { still has a diagnosis of osteoporosis }\end{array}$ \\
\hline Bone density matters & $\begin{array}{l}\text { Greater increases of bone density with treatment are associated with larger reductions in fracture } \\
\text { risk }\end{array}$ \\
\hline Fracture is a "bone attack" & $\begin{array}{l}\text { A recent fracture is associated with high risk of another fracture in the next few years ("imminent } \\
\text { fracture risk"); urgent medical attention is needed }\end{array}$ \\
\hline Anabolic therapy builds new bone & $\begin{array}{l}\text { All approved drugs for osteoporosis can increase bone density and reduce fracture risk, but only } \\
\text { anabolic drugs improve bone structure }\end{array}$ \\
\hline $\begin{array}{l}\text { Treat to achieve an acceptable level } \\
\text { of fracture risk ("treat-to-target") }\end{array}$ & $\begin{array}{l}\text { Initial treatment and treatment changes should consider the liklihood of achieving an acceptable } \\
\text { level of fracture risk; T-score is currently the most useful surrogate for fracture risk in treated } \\
\text { patients }\end{array}$ \\
\hline
\end{tabular}

A phase 3 clinical trial (FRAME) evaluated the efficacy and safety of romosozumab in 7,180 postmenopausal women with osteoporosis (2). It was found that 12 months of treatment with romosozumab $210 \mathrm{mg}$ subcutaneously (SC) every 1 month $(\mathrm{Q} 1 \mathrm{M})$ significantly reduced the risk of vertebral fractures and clinical fractures compared with placebo. When women in the romosozumab and placebo groups were transitioned to receive denosumab, a potent antiresorptive agent, for the following year, the difference in vertebral fracture rates persisted, with fewer vertebral fractures in the romosozumab-to-denosumab group compared with placebo-to-denosumab. In a phase 3 study of romosozumab in men with osteoporosis (BRIDGE), changes in BMD and bone turnover markers were similar to what was observed with women in FRAME, suggesting that fracture risk reduction may be similar as well (3). Another phase 3 study (ARCH), evaluated the efficacy and safety of romosozumab for 1 year followed by alendronate for 1 year compared with alendronate for 2 years in 4,093 postmenopausal women with osteoporosis (4). The risk of vertebral fractures was significantly lower in the romosozumab-to-alendronate group than alendronateto-alendronate group. In clinical practice, it may be recognized that a patient being treated with alendronate is responding poorly or at higher risk of fracture than previously known, leading to consideration of switching to anabolic therapy. This scenario was assessed in a phase 3 trial (STRUCTURE) in which 436 postmenopausal women treated with alendronate for at least 3 years (mean 6.2 years) were randomized to receive romosozumab or teriparatide for the next 12 months (5). BMD increased significantly at the femoral neck and total hip with romosozumab but not with teriparatide $(\mathrm{P}<0.0001)$; $\mathrm{BMD}$ increased lumbar spine with both anabolic agents, but significantly greater with romosozumab $(\mathrm{P}<0.0001)$. Since greater increases of $\mathrm{BMD}$ with treatment are associated with greater reductions in fracture risk (6), it may be preferable to switch from a bisphosphonate to romosozumab rather than teriparatide in appropriately selected high risk patients.

The dose of romosozumab used in the phase 3 trials and approved for clinical use (210 $\mathrm{mg}$ Q1M) was established with a 12 month phase 2 dose ranging study in 419 postmenopausal women with low BMD (7). Women enrolled in this study were randomized to receive 1 of 5 different doses of romosozumab $(70 \mathrm{mg}, 140 \mathrm{mg}$, or 210 Q1M, or $140 \mathrm{mg}$ or $210 \mathrm{mg}$ Q3M), placebo injections, or 1 
of 2 open label active comparators-oral alendronate $(70 \mathrm{mg}$ weekly) or teriparatide ( $20 \mathrm{mcg}$ daily). The primary endpoint was lumbar spine BMD at 12 months. All doses of romosozumab increased BMD at the lumbar spine compared with a $0.1 \%$ decrease with placebo. There was an $11.3 \%$ BMD increase with the $210 \mathrm{mg}$ Q1M dose of romosozumab, $4.1 \%$ increase with alendronate, and $7.1 \%$ increase with teriparatide. Transient increases in bone formation markers and persistent decreases in a bone resorption marker were observed.

A report by McClung et al. (8) described the findings of continuation of the 12 -month phase 2 study for an additional 12 months, followed by a 12 -month denosumab extension phase. After the first 12 months, women in the romosozumab group continued with their assigned doses for the next 12 months, while those taking alendronate were switched to receive romosozumab $140 \mathrm{mg}$ Q1M, and those taking teriparatide were disenrolled from the study. After a total of 24 months of study participation, eligible consenting women were randomized in a $1: 1$ ratio to receive denosumab $60 \mathrm{mg}$ SC Q6M or placebo SC Q6M for an additional 12 months (total of 36 months study participation). At 24 months, the largest BMD increase ( $15.1 \%$ at the lumbar spine compared with baseline) was observed with romosozumab $210 \mathrm{mg}$ Q1M. In women transitioned from romosozumab $210 \mathrm{mg}$ Q1M for 24 months to denosumab for the next 12 months, there were further increases in BMD, with a total increase of $19.4 \%$ at the lumbar spine and $7.1 \%$ at the total hip. For women receiving romosozumab $210 \mathrm{mg}$ Q1M for 24 months followed by placebo for the next 12 months, there was a decrease in BMD toward pretreatment levels.

The totality of the evidence with romosozumab and other therapeutic agents for treating osteoporosis is helpful in the challenging task of discussing with patients the expected benefits and potential risks of treatment. Common questions from patients who are advised to start treatment, and considerations for responding, are as follows.

\section{What drug should I take?}

The initial drug choice is dependent on considerations that include baseline BMD, prior fractures, level of fracture risk, patient preference, expected adherence to therapy, previous drug experiences, comorbidities, cost, and insurance coverage. A concept that may help with choosing initial therapy, or changing therapy after it is started, is "treat-totarget" ("goal-directed treatment") (9), whereby treatment is selected according to the likelihood of achieving an acceptable level of fracture risk. This is different than the more conventional strategy of starting treatment for all patients with a generic oral bisphosphonate, the least expensive of treatment options, unless there is a contraindication. Considering accumulating evidence that greater increases in BMD are associated with better reduction of fracture risk (6) and reports that increasing T-score to about -2.0 to -1.5 is associated with optimal reduction of fracture risk, at least for denosumab (10), BMD (expressed as T-score) may be the most useful treatment target. More investigation is needed to fully validate the clinical utility of this concept with all therapeutic agents.

For patients at high risk of fracture, clinical trials, including ARCH, have shown that anabolic therapy is superior to antiresorptive therapy for reduction of fracture risk (4,11-13). The sequence of therapy is also important, with greater benefit for high risk patients when treatment is started with an anabolic agent rather than beginning with antiresorptive therapy and later switching to anabolic (14). A good example of the benefit of anabolic followed by antiresorptive therapy is the "foundation effect" described in a report showing that treatment with romosozumab for 1 year followed by denosumab for 1 year increases BMD to a level comparable to about 7 years of treatment with denosumab alone (15). For a patient at high risk of fracture, especially one with a recent fracture (16), initiating treatment with an anabolic agent is an attractive option.

\section{What are the side effects?}

All medications, and virtually every human activity, have possible adverse effects. Drugs for osteoporosis are no exception. The US Food and Drug Administration (FDA) requires that the label for each of the anabolic agents includes a boxed warning. For teriparatide and abaloparatide the warning is for potential risk of osteosarcoma due to uncertain relevance of these drugs having a dose-dependent increase in the incidence of osteosarcoma in rats. The recommended remedy is to avoid these agents in patients at high baseline risk for osteosarcoma, such as those with unexplained elevation of serum alkaline phosphatase, Paget's disease of bone, or radiation therapy involving the skeleton. It is reassuring to note that there is no evidence of increased risk of osteosarcoma in humans in a clinical review of 10 years of teriparatide experience and 6 years of $\mathrm{PTH}(1-84)$ (17) and no evidence of increased risk in the first 8 years of a 
teriparatide patient registry with an estimated 242,782 person-years of observation (18). Less is known about abaloparatide, which is also a PTH-derived product, due to fewer years of clinical use, but it is likely that the risk is also very low, similar to teriparatide.

For romosozumab, the warning is for potential risk of myocardial infarction, stroke, and cardiovascular death due to uncertainty in clinical trials. The recommended remedy is to avoid the treatment with romosozumab in patients who have had a myocardial infarction or stroke in the past 1 year and to stop treatment if a patient has a myocardial infarction or stroke while receiving romosozumab. In FRAME (2), the largest clinical trial with romosozumab, there was no difference in cardiovascular endpoints with romosozumab compared with placebo. Likewise, in the first 24 months of the phase 2 clinical trial of romosozumab, adverse effects and serious adverse effects were similar in the placebo groups and all treatment groups, with comparable risk of cardiovascular endpoints (8). However, in ARCH (4) there was a numerical increase in adjudicated cardiovascular adverse events after 12 months of treatment with romosozumab $(2.5 \%)$ compared with alendronate $(1.9 \%)$. There is no plausible mechanism of action to explain possible adverse cardiovascular effects with romosozumab. It is not known whether the findings in ARCH are due to a real increase of risk with romosozumab, a protective of effect of alendronate, or a statistical aberration. It should be noted that for all 3 anabolic agents, these are warnings, not contraindications, since there must be known harm to be categorized as a contraindication. The boxed warnings should be considered when evaluating the balance of benefits and risks for each patient.

With bisphosphonates, there have been reports of rare occurrences of osteonecrosis of the jaw (ONJ) and atypical femur fractures (AFF). Media attention describing these events often does not communicate their rarity compared with the benefits (19). Media reports can be frightening to patients, sometimes resulting in discontinuation or failure to start treatment for osteoporosis, potentially leading more fractures and deaths (20). Some of the same concerns apply to denosumab, which has also been rarely associated with $\mathrm{ONJ}$ and AFF. Effective communication of the balance of benefits (fewer fractures) and risk of possible side effects (e.g., ONJ and AFF) is a skill that can be acquired (21), often with the use of decision aids and comparison with daily activities, such as driving a car, associated with much higher risk. Regrettably, health care providers may have insufficient time, interest, or skills to have such a conversation.

\section{How long do I have to take it?}

Osteoporosis is a lifelong disease that warrants lifelong attention, as with other common chronic diseases, such as hypertension, diabetes, and hypercholesterolemia. There is no temporary treatment, whether it be with drugs or lifestyle modification, for a lifelong disease. Once a diagnosis of osteoporosis is made, the diagnosis persists, even if treatment results in improvement of T-score to $>-2.5$ (22). Changing the diagnosis to osteopenia or normal after effective treatment is given may have adverse clinical consequences if needed treatment is discontinued or changed, or insurance coverage for treatment is withdrawn.

There is a common misperception that all osteoporosis drugs have a time limit, whereas time limits apply only to the 3 approved anabolic agents. All drugs stop working when they are stopped. The non-bisphosphonates stop working very quickly. This is well illustrated in the phase 2 trial of romosozumab, where patients transitioned from 2 years of romosozumab to placebo for the next 12 months were reported to have a loss of BMD (8). Stopping denosumab, a non-bisphosphonate with a robust antiresorptive effect, is also followed by loss of BMD (23), and a rapid return of vertebral fracture risk to baseline (24). The theme of these studies and others is that stopping nonbisphosphonate therapy in patients at high risk of fracture should be quickly followed by another therapeutic agent; if this is not done, benefits will be rapidly lost.

Bisphosphonates, due to long skeletal half-life, may have persistence of antiresorptive effects and anti-fracture benefit for a limited period of time after stopping. This has led to the concept of a "drug holiday" for patients who have had long-term bisphosphonate therapy and are no longer at high risk of fracture (25), based on the rationale that temporary withholding of a bisphosphonate might allow for persistent benefits, at least for a while, with a reduction of the risk of ONJ and AFF. Such patients must be monitored and treatment resumed when fracture risk is high.

\section{Does it build new bone?}

It is common for patients to confuse building new bone with increasing BMD. A useful message is that all approved osteoporosis drugs can increase BMD, improve bone strength, and reduce fracture risk, but only the anabolic agents build new bone and restore, at least partially, 
degraded bone structure. The use of bone images and models can be helpful for communicating this concept to patients. When patients have a better understanding of the actions of anabolic therapy, they are better equipped to make a treatment decision.

\section{Acknowledgments}

Funding: None.

\section{Footnote}

Conflicts of Interest: The author has completed the ICMJE uniform disclosure form (available at http://dx.doi. org/10.21037/atm.2020.03.196). EML has no direct income from potentially conflicting entities. His employer, New Mexico Clinical Research \& Osteoporosis Center, has received research grants from Radius, Amgen, Mereo, Bindex; income for service on scientific advisory boards or consulting for Amgen, Radius, Alexion, Sandoz, Samsung Bioepis, Sanifit; service on speakers' bureaus for Radius, Alexion; project development for University of New Mexico; and royalties from UpToDate for sections on DXA, fracture risk assessment, and prevention of osteoporosis. He is a board member of the National Osteoporosis Foundation, International Society for Clinical Densitometry, and Osteoporosis Foundation of New Mexico.

Ethical Statement: The author is accountable for all aspects of the work in ensuring that questions related to accuracy or integrity of any part of the work are appropriately investigated and resolved.

Open Access Statement: This is an Open Access article distributed in accordance with the Creative Commons Attribution-NonCommercial-NoDerivs 4.0 International License (CC BY-NC-ND 4.0), which permits the noncommercial replication and distribution of the article with the strict proviso that no changes or edits are made and the original work is properly cited (including links to both the formal publication through the relevant DOI and the license). See: https://creativecommons.org/licenses/by-nc-nd/4.0/.

\section{References}

1. International Osteoporosis Foundation. Facts and statistics 2020 February 21, 2020. Available online: https://www. iofbonehealth.org/facts-statistics\#category-14

2. Cosman F, Crittenden DB, Adachi JD, et al. Romosozumab Treatment in Postmenopausal Women with Osteoporosis. N Engl J Med 2016;375:1532-43.

3. Lewiecki EM, Blicharski T, Goemaere S, et al. A Phase III Randomized Placebo-Controlled Trial to Evaluate Efficacy and Safety of Romosozumab in Men With Osteoporosis. J Clin Endocrinol Metab 2018;103:3183-93.

4. Saag KG, Petersen J, Brandi ML, et al. Romosozumab or Alendronate for Fracture Prevention in Women with Osteoporosis. N Engl J Med 2017;377:1417-27.

5. Langdahl BL, Libanati C, Crittenden DB, et al. Romosozumab (sclerostin monoclonal antibody) versus teriparatide in postmenopausal women with osteoporosis transitioning from oral bisphosphonate therapy: a randomised, open-label, phase 3 trial. Lancet 2017;390:1585-94.

6. Bouxsein ML, Eastell R, Lui LY, et al. Change in Bone Density and Reduction in Fracture Risk: A MetaRegression of Published Trials. J Bone Miner Res 2019;34:632-42.

7. McClung MR, Grauer A, Boonen S, et al. Romosozumab in postmenopausal women with low bone mineral density. N Engl J Med 2014;370:412-20.

8. McClung MR, Brown JP, Diez-Perez A, et al. Effects of 24 Months of Treatment With Romosozumab Followed by 12 Months of Denosumab or Placebo in Postmenopausal Women With Low Bone Mineral Density: A Randomized, Double-Blind, Phase 2, Parallel Group Study. J Bone Miner Res 2018;33:1397-406.

9. Cummings SR, Cosman F, Lewiecki EM, et al. GoalDirected Treatment for Osteoporosis: A Progress Report From the ASBMR-NOF Working Group on GoalDirected Treatment for Osteoporosis. J Bone Miner Res 2017;32:3-10.

10. Ferrari S, Libanati C, Lin CJF, et al. Relationship Between Bone Mineral Density T-Score and Nonvertebral Fracture Risk Over 10 Years of Denosumab Treatment. J Bone Miner Res 2019;34:1033-40.

11. Saag KG, Shane E, Boonen S, et al. Teriparatide or alendronate in glucocorticoid-induced osteoporosis. $\mathrm{N}$ Engl J Med 2007;357:2028-39.

12. Hadji P, Zanchetta JR, Russo L, et al. The effect of teriparatide compared with risedronate on reduction of back pain in postmenopausal women with osteoporotic vertebral fractures. Osteoporos Int 2012;23:2141-50.

13. Kendler DL, Marin F, Zerbini CAF, et al. Effects of teriparatide and risedronate on new fractures in post- 
menopausal women with severe osteoporosis (VERO): a multicentre, double-blind, double-dummy, randomised controlled trial. Lancet 2018;391:230-40.

14. Cosman F, Nieves JW, Dempster DW. Treatment Sequence Matters: Anabolic and Antiresorptive Therapy for Osteoporosis. J Bone Miner Res 2017;32:198-202.

15. Cosman F, Crittenden DB, Ferrari S, et al. FRAME Study: The Foundation Effect of Building Bone With 1 Year of Romosozumab Leads to Continued Lower Fracture Risk After Transition to Denosumab. J Bone Miner Res 2018;33:1219-26.

16. Johansson H, Siggeirsdottir K, Harvey NC, et al. Imminent risk of fracture after fracture. Osteoporos Int 2017;28:775-80.

17. Cipriani C, Irani D, Bilezikian JP. Safety of osteoanabolic therapy: a decade of experience. J Bone Miner Res 2012;27:2419-28.

18. Gilsenan A, Harding A, Kellier-Steele N, et al. The Forteo Patient Registry linkage to multiple state cancer registries: study design and results from the first 8 years. Osteoporos Int 2018;29:2335-43.

19. Cipriani C, Pepe J, Minisola S, et al. Adverse effects of media reports on the treatment of osteoporosis. J Endocrinol Invest 2018;41:1359-64.

20. Sambrook PN, Chen JS, Simpson JM, et al. Impact of

Cite this article as: Lewiecki EM. Romosozumab, clinical trials, and real-world care of patients with osteoporosis. Ann Transl Med 2020;8(15):974. doi: 10.21037/atm.2020.03.196 adverse news media on prescriptions for osteoporosis: effect on fractures and mortality. Med J Aust 2010;193:154-6.

21. Lewiecki EM. The role of risk communication in the care of osteoporosis. Curr Osteoporos Rep 2011;9:141-8.

22. Lewiecki EM, Binkley N, Bilezikian JP. Treated Osteoporosis Is Still Osteoporosis. J Bone Miner Res 2019;34:605-6.

23. Miller PD, Bolognese MA, Lewiecki EM, et al. Effect of denosumab on bone density and turnover in postmenopausal women with low bone mass after longterm continued, discontinued, and restarting of therapy: A randomized blinded phase 2 clinical trial. Bone 2008;43:222-9.

24. Cummings SR, Ferrari S, Eastell R, et al. Vertebral Fractures After Discontinuation of Denosumab: A Post Hoc Analysis of the Randomized Placebo-Controlled FREEDOM Trial and Its Extension. J Bone Miner Res 2018;33:190-8.

25. Adler RA, El-Hajj Fuleihan G, Bauer DC, et al. Managing Osteoporosis in Patients on Long-Term Bisphosphonate Treatment: Report of a Task Force of the American Society for Bone and Mineral Research. J Bone Miner Res 2016;31:16-35. 